\title{
Erasure and Polymorphism in Pure Type Systems
}

\author{
Nathan Mishra-Linger and Tim Sheard \\ Portland State University \\ $\{$ rlinger, sheard $\} @$ cs.pdx.edu
}

\begin{abstract}
We introduce Erasure Pure Type Systems, an extension to Pure Type Systems with an erasure semantics centered around a type constructor $\forall$ indicating parametric polymorphism. The erasure phase is guided by lightweight program annotations. The typing rules guarantee that well-typed programs obey a phase distinction between erasable (compile-time) and non-erasable (run-time) terms.

The erasability of an expression depends only on how its value is used in the rest of the program. Despite this simple observation, most languages treat erasability as an intrinsic property of expressions, leading to code duplication problems. Our approach overcomes this deficiency by treating erasability extrinsically.

Because the execution model of EPTS generalizes the familiar notions of type erasure and parametric polymorphism, we believe functional programmers will find it quite natural to program in such a setting.
\end{abstract}

\section{Background and Motivation}

Statically typed programming languages have evolved ever more expressive type systems. The drive towards increased expressiveness inevitably leads to dependent types, a proven technology for verifying strong correctness properties of real programs 15958 . For this reason, researchers have long sought practical ways to include dependent types in programming languages.

Heavy use of the expressive power of dependent types involves the embedding of proofs of program properties into the program text itself. Often, such proofs play no essential part in the execution of the program. They are necessary only as evidence used by the type-checker. Because these proofs can be quite large, an erasure semantics is critical for practical implementation of a dependently typed programming language.

However, proofs are not the only erasable parts of a program. Any time a program exhibits parametric polymorphism (whether it be polymorphism over proofs, types, numbers, or any other type of value) there are portions of the program that should be erased. One thesis of this paper is that parametric polymorphism can be understood entirely in terms of erasability.

R. Amadio (Ed.): FOSSACS 2008, LNCS 4962, pp. 350-364, 2008.

(C) Springer-Verlag Berlin Heidelberg 2008 


\subsection{Erasability Is Extrinsic Rather than Intrinsic}

Which parts of a progran 1 may be erased in an erasure-semantics? Our investigation of erasure semantics is grounded in a simple observation: Erasability of a program expression is not a property of the expression itself but rather a property of the context in which we find it. Erasability of a program expression is determined not by what it is, but by how it is used. In other words, erasability is an extrinsic rather than an intrinsic property. We give several examples demonstrating this principle.

Type annotations. The domain annotation $A$ in the $\beta$ rule,

$$
(\lambda x: A . M) N \rightarrow_{\beta} M[N / x]
$$

is simply discarded. For this reason, we may safely erase the domain annotations of all $\lambda$-abstractions in a program without changing their computational behavior. In this case, the context in which $A$ appears determines its erasability.

Dummy $\lambda$-binders. The erasure of domain annotations may cause some $\lambda$ binders to become superfluous. Consider the term ( $\lambda \alpha$ :Type. $\lambda x: \alpha . x)$ Nat 5 . After erasing type annotations, we are left with $(\underline{\lambda \alpha} . \lambda x . x) \underline{N a t} 5$, in which the binder $\lambda \alpha$ is superfluous because $\alpha$ no longer appears anywhere in its scope. For any such dummy binder $\lambda x$, the resulting specialized $\beta$ rule

$$
(\lambda x . M) N \rightarrow \rightarrow_{\beta} M \quad \text { if } x \notin F V(M)
$$

discards both the dummy binder and the argument which it would otherwise bind to $x$. Therefore we may erase both the binding site $\lambda x$ and any argument $N$ at an application site to which this $\lambda$-abstraction may flow during program execution. By this reasoning, we may erase the underlined portions of our previous example term, resulting in $(\lambda x . x) 5$.

However, other $\lambda$-abstractions may flow to some of those same application sites. We should not erase the argument $N$ at an application site $M @ N$ unless every $\lambda$-abstraction that may flow to be the value of $M$ has a dummy binder. In general, the "may-flow-to" relation induces a bipartite graph (see Figure 1). In order to decide if a given $\lambda$-binder or @-argument may be safely erased, we must analyze its entire connected component (CC) in this $\lambda / @$ graph.

In this type of erasure step, the usage of a term determines its erasability. The (local) erasability of a binder $\lambda x$ depends on how $x$ is (or is not) used in its scope. The erasability of an argument $N$ depends on its context - whether the function that is applied to it always ends up being a $\lambda$-abstraction with a dummy binder.

\footnotetext{
${ }^{1}$ For our purposes here, a program is a term in a typed $\lambda$-calculus.

${ }^{2}$ We sometimes write @ for application in order to have a more tangible notation than mere juxtaposition.
} 


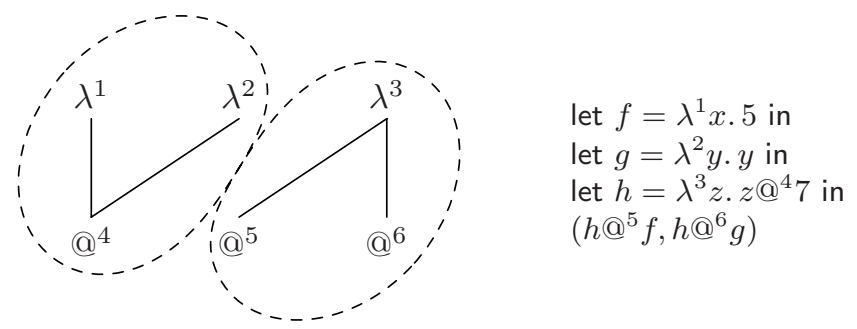

Fig. 1. The $\lambda / @$ graph induced by the "may-flow-to" relation of a simple program. The use of $y$ in $\lambda^{2}$ 's body prevents erasure of both the $@^{4}$-argument and the $\lambda^{1}$-binder.

Cascading Erasure. Erasure of @-arguments may make other $\lambda$-binders into dummies, thereby enabling erasure in other CCs of the $\lambda / @$ graph. Consider the following family of identity functions.

\begin{tabular}{|c|c|c|}
\hline $\begin{array}{l}\mathrm{t} i d_{0}=\lambda a: s \cdot \lambda x: a \cdot x \text { in } \\
\mathrm{t} i d_{1}=\lambda a: s \cdot \lambda x: a \cdot i d_{0} \text { a } x \text { in } \\
\mathrm{t} i d_{2}=\lambda a: s \cdot \lambda x: a \cdot i d_{1} \text { a } x \text { in } \\
\mathrm{t} i d_{3}=\lambda a: s \cdot \lambda x: a \cdot i d_{2} \text { a } x \text { in }\end{array}$ & $\Rightarrow$ & $\begin{array}{l}\text { let } i d_{0}=\lambda a \cdot \lambda x \cdot x \text { in } \\
\text { let } i d_{1}=\lambda a \cdot \lambda x \cdot i d_{0} \quad a x \text { in } \\
\text { let } i d_{2}=\lambda a \cdot \lambda x \cdot i d_{1} \quad a x \text { in } \\
\text { let } i d_{3}=\lambda a \cdot \lambda x \cdot i d_{2} \quad a x \text { in }\end{array}$ \\
\hline
\end{tabular}

After the initial erasure of domain annotations, a cascading sequence of $\lambda / @$ erasure steps is possible in this program. (Consider the $\lambda a$ binders).

\subsection{Intrinsic Notions of Erasability Beget Code Duplication}

Most prior attempts to combine dependent types and erasure semantics treat erasability as an intrinsic property. These attempts may be divided into two categories: erasure first and dependent types first.

Erasure first. Languages in this category start with a commitment to erasure semantics in the form of a syntactic phase distinction whereby types and program values may not depend on each other computationally. Singleton types are then used to simulate dependently typed programming. Examples of this approach include Dependent ML [22], $\Omega$ mega 20]19], Applied Type Systems 17, and Haskell with generalized algebraic datatypes [16].

Singleton types are type families $T: I \rightarrow$ Type for which each type index $i: I$ uniquely determines the one value of type $T i$. For example, the declarations

$$
\begin{aligned}
\text { datakind } N a t \uparrow & : \text { kind } \\
\text { where } Z \text { Zero } \uparrow & : \text { Nat } \uparrow \\
\text { Succ } \uparrow & : \text { Nat } \uparrow \rightarrow N a t \uparrow
\end{aligned}
$$

$$
\begin{aligned}
& \text { datatype Nat! : Nat } \rightarrow \text { Type } \\
& \text { where Zero! : Nat! Zero } \uparrow \\
& \text { Succ! : Nat! } n \rightarrow \text { Nat! }(\text { Succ } \uparrow n)
\end{aligned}
$$

introduce a singleton type family for the naturals. The datakind declaration defines a copy of the natural numbers at the type-level. The singleton type Nat! then connects the type-level version Nat个 to the level of run-time expressions. 
A singleton type acts as a proxy between run-time and compile-time notions of the same datatype: natural numbers in this case. Whenever a program does case analysis on the value of a singleton type, the type-checker benefits from the same case analysis at the type-level. In this way, dependence of types on values is simulated.

Dependent types first. Languages in this category start with full dependent types. An erasure phase then strips out parts of the program that are irrelevant to its run-time execution. Examples of this approach include Cayenne [3], Coq [1], and Epigram [126].

In Cayenne and Coq, the erasability of a subterm depends on its type. All types (subterms of type Type) are erased in Cayenne and Epigran3. Coq's program extraction mechanism supports erasure of proofs as well as types. A proof is distinguished by having a proposition as its type, and propositions are distinguished as terms of type Prop. In contrast to the universe Prop, Coq has another universe Set that is the type of the types of all non-erasable program terms.

Code duplication. Because languages in both categories treat erasability as an intrinsic property of an expression, usually determined by its type, users of these languages are sometimes forced to duplicate definitions of datatypes and functions over them in order to achieve a desired erasure behavior. In the erasure-first approach, programming with singleton types requires duplication of datatype definitions at the "type" and "kind" levels of the type hierarchy 4 , as well as duplication of functions that operate on them. In the dependent-typesfirst approach, duplication of datatypes is also required if we want values of a particular type to be erased in one part of a program but not in another.

\subsection{Methodology and Outline}

We treat erasability as a property not of a term itself, but of the context in which it is used. In $\lambda$-calculus, functions reify such contexts, so we track erasability as a property of functions by distinguishing between functions that do not depend computationally on their arguments (of type $\forall x: A . B$ ) and those that might (of type $\Pi x: A . B)$.

Note that the same $A$ is used in both cases, because erasability is no longer an intrinsic property of $x$, but rather a property of the (functional) contexts making use of $x$. In this way we avoid the code duplication problem. We have one type $A$ and therefore functions over $A$ can be written once.

Pure Type Systems (PTS) are a well-known family of typed $\lambda$-calculi that encompass a wide variety of type systems [4]. Most dependently typed languages

\footnotetext{
${ }^{3}$ Some work on representations of inductive types in Epigram notes that values of type families need not store certain indices that, regardless of their type, are uniquely determined by the value's data constructor.

${ }^{4}$ The singleton type itself may be thought of as a maximally informative copy of the original datatype.
} 
have a PTS at their core. Therefore PTS is a good setting for studying features of dependently types languages. Section 2 briefly reviews the basics of PTS.

Section 3 introduces a conservative extension to PTS called Erasure Pure Type Systems (EPTS) supporting the $\forall$ type described above and rules for checking that programs using this type satisfy a phase distinction.

Section 4 introduces another PTS variant called Implicit Pure Type Systems (IPTS) that serves as the target language of the erasure phase. This language is very closely related to Miquel's Implicit Calculus of Constructions [13.

Section 5 introduces our erasure translation from EPTS to IPTS. This operation is the basis for the erasure semantics of EPTS (Section 6). We prove that erasure exhibits properties one would expect: It respects the static and dynamic semantics of programs and eliminates portions of the source program that do not affect its final value.

In Sections 7, 8, and 9 we discuss implementation issues, future work, and our conclusions.

\section{Pure Type Systems}

Pure Type Systems bring organization to type theory [4. They generalize Barendregt's $\lambda$-cube, which includes such familiar systems as the simply typed $\lambda$ calculus, Systems F and $F^{\omega}$, the Edinburgh Logical Framework, and the Calculus of Constructions.

Pure Type Systems are a family of typed lambda calculi. Each PTS has a specification $(\mathcal{S}, \mathcal{A}, \mathcal{R})$ consisting of a set $\mathcal{S}$ of sorts (a.k.a. universes), a set $\mathcal{A} \subseteq \mathcal{S}^{2}$ of axioms, and a set $\mathcal{R} \subseteq \mathcal{S}^{3}$ of rules. We assume a fixed specification throughout the development. The syntax of PTS is as follows:

$$
M, N, A, B::=x|\lambda x: A . M| M N|\Pi x: A . B| s
$$

Note that there is a single syntactic category for types and terms. The metavariable $s$ is used to denote sorts. The typing rules of PTS are parameterized by $\mathcal{A}$ and $\mathcal{R}$ and can be obtained from those of EPTS (which we will discuss shortly) by simply ignoring all erasure annotations.

\section{Erasure Pure Type Systems}

This section introduces Erasure Pure Type Systems (EPTS), an extension of Pure Type Systems (PTS) with annotations indicating erasable parts of a program. The EPTS type system checks the erasability of the parts so annotated.

Syntax. The syntax of EPTS is that of PTS with erasure annotations added.

$$
\begin{aligned}
& \text { (terms) } \quad M, N, A, B::=x\left|\lambda^{\tau} x: A . M\right| M @^{\tau} N\left|\Pi^{\tau} x: A . B\right| s \\
& \text { (contexts) } \quad \Gamma, \Delta::=\varepsilon \mid \Gamma, x:^{\tau} A \\
& \text { (times) } \tau::=\mathrm{r} \mid \mathrm{c}
\end{aligned}
$$


The metavariable $\tau$ ranges over erasure annotations. The annotation $r$ means "run-time". Syntax with this annotation behaves just as it would in PTS without any annotation. The annotation c means "compile-time" and indicates erasable portions of a program.

All $\Pi \mathrm{s}, \lambda \mathrm{s}$, and @ $@_{\mathrm{s}}$ are annotated. Annotations on $\Pi \mathrm{s}$ distinguish between computational dependence $\left(\Pi^{\mathrm{r}}\right)$ and polymorphism $\left(\Pi^{\mathrm{C}}\right)$. In concrete syntax, we would simply write $\Pi$ for $\Pi^{\mathrm{r}}$ and $\forall$ for $\Pi^{\mathrm{C}}$, but this choice of abstract syntax affords us economy of presentation. These annotations guide the erasure operation to be defined in Section 5.

Type System. Figure 2 contains typing rules for EPTS. There are two forms of judgment, $\Gamma \vdash M:{ }^{\mathrm{C}} A$ and $\Gamma \vdash M:^{r} A$. The judgment $\Gamma \vdash M:^{r} A$ says that $M$ is a well-formed run-time entity, while $\Gamma \vdash M:^{\mathrm{C}} A$ says that $M$ is a well-formed compile-time (erasable) entity.

The type system needs to check that all $\lambda \mathrm{s}$ and @ $@_{\mathrm{s}}$ marked c are erasable. Recall from Section 1.1 that erasability of $\lambda_{\mathrm{s}}$ and @s in the $\lambda /$ @ graph must be considered one connected component (CC) at a time. The flow analysis implicit in the typing rules ensures that every $\lambda$ and @ in the same $\mathrm{CC}$ are annotated with the same $\tau$. Therefore, if every $\lambda^{\mathrm{C}}$-binder is erasable, then so is every $\mathrm{C}^{\mathrm{C}}$ argument. So we need only verify that each $\lambda^{\mathrm{C}}$ is erasable - for each $\lambda^{\mathrm{C}} x: A . M$ in the program, all free occurrences of $x$ in $M$ must appear either inside a type annotation or inside an $\Theta^{\mathrm{C}}$ argument.

The typing rules enforce this invariant using the following technique, due to Pfenning [17. Each $\lambda^{\mathrm{C}}$-bound variable $x$ is marked with $\mathrm{c}$ when it is added to the typing context. This mark is then locally switched off (reset) whenever we check a type annotation or $\Theta^{\mathrm{c}}$ argument. We then require that the mark $\mathrm{c}$ has been switched off by the time we reach any occurrence of $x$. For economy of presentation, an "off" mark in the typing context is represented as an $r$ mark. Passing this mark/reset/check test guarantees that each $\lambda^{\mathrm{C}}$ is actually erasable.

\section{Definition (Context Reset Operation) $\Gamma^{\circ}$}

$$
\varepsilon^{\circ}=\varepsilon \quad\left(\Gamma, x:^{\tau} A\right)^{\circ}=\Gamma^{\circ}, x:^{r} A
$$

The key steps of the mark/reset/check test are found in the typing rules $\Pi$ Intro (mark), $\Pi$-ELIM and RESET (reset), and VAR (check). In particular, notice how rule $\Pi$-INTRO marks context entries and $\Pi$-ELIM checks function arguments for both $\tau=\mathrm{r}$ and $\tau=\mathrm{c}$.

Rules VAR, WEAK, $\Pi$-InTro, and CONV each have a premise of the form $\Gamma \vdash A:{ }^{\mathrm{C}} s$. The purpose of these rules is to check that $A$ is well-formed as a type. Because these rules deal explicitly with types, they use the compile-time typing judgment. In particular, domain annotations are considered as compiletime entities in the $\Pi$-InTro rule.

The $\Pi$-Form rule may seem counter-intuitive at first. Because $\Pi$ is a type former, one might expect this rule to use c-judgments rather than $r$ ones. However, in a dependently typed language, terms may compute (at run-time) to 
$\Gamma \vdash M:^{\tau} A$

$\begin{array}{lll}\text { Axiom } & \text { VAR } & \text { WEAK } \\ \frac{\left(s_{1}, s_{2}\right) \in \mathcal{A}}{\vdash s_{1}:^{\mathrm{r}} s_{2}} & \frac{\Gamma \vdash A:^{\mathrm{c}} s}{\Gamma, x:^{\mathrm{r}} A \vdash x:^{\mathrm{r}} A} & \frac{\Gamma \vdash A{ }^{\mathrm{c}} s \quad \Gamma \vdash M:^{\mathrm{r}} B}{\Gamma, x:^{\tau} A \vdash M:^{\mathrm{r}} B}\end{array}$

\section{II-Form}

$\frac{\left(s_{1}, s_{2}, s_{3}\right) \in \mathcal{R} \quad \Gamma \vdash A:^{\mathrm{r}} s_{1} \quad \Gamma, x:^{\mathrm{r}} A \vdash B:^{\mathrm{r}} s_{2}}{\Gamma \vdash \Pi^{\tau} x: A \cdot B:^{\mathrm{r}} s_{3}}$

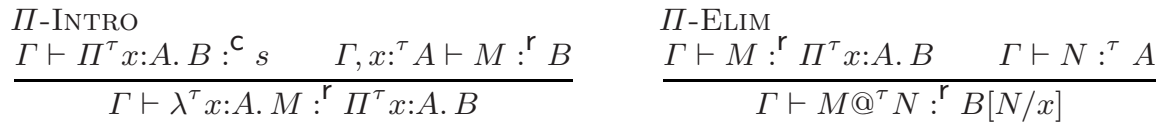

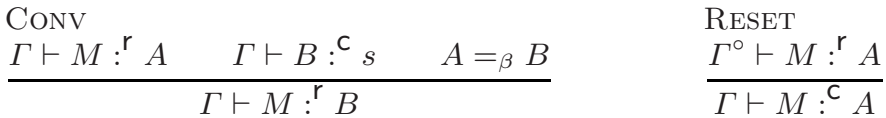

Fig. 2. Typing rules for EPTS. (The typing rules for PTS may be obtained from these by ignoring all erasure annotations and removing the then useless RESET rule).

types, so the $r$ is appropriate. Another possible surprise is that $x$ is marked with $r$ rather than $\tau$ in the typing context of $B$. This is because the binding site of the $x$ will never be erased: The only purpose of the context mark $\mathrm{c}$ is to check erasability of a $\lambda^{\mathrm{c}}$.

If we ignore erasure annotations, these typing rules are exactly those of PTS. The extra restrictions on erasure annotations ensure the following sort of phase distinction: evaluation of any well-typed term never depends on its compile-time portions. We formalize and prove this in Section 5 .

Semantics. The default operational semantics of EPTS is simply $\beta$-reduction. We do not commit to any particular evaluation order, so the single-step reduction relation is non-deterministic.

Actually this is only one of two different operational semantics for EPTS. The remainder of this paper introduces an erasure semantics with potential for more efficient execution.

Meta-theory. The top half of Figure 4 depicts the meta-theory of EPTS. Each box in that figure contains a particular result of the meta-theory. As the development follows closely that of Pure Type Systems, we focus on the changes due to introducing erasure annotations.

First we investigate properties of the context reset operation $\Gamma^{\circ}$. It is idempotent (Lemma 1) and weakens the strength of the typing assumptions (Lemma 2). An admissible phase-weakening rule (Corollary 3 ) follows immediately from Lemma 2. Proofs: Lemma 1 is easily proved by induction on $\Gamma$. Lemma 2 is proved by structural induction on the typing derivation. The interesting cases 
$\Gamma \vdash M: A$

$$
\begin{array}{lll}
\begin{array}{l}
\text { Axiom } \\
\left(s_{1}, s_{2}\right) \in \mathcal{A}
\end{array} & \begin{array}{l}
\text { VAR } \\
\vdash s_{1}: s_{2}
\end{array} \quad \frac{\Gamma \vdash A: s}{\Gamma, x: A \vdash x: A} & \frac{\Gamma \vdash A: s \quad \Gamma \vdash M: B}{\Gamma, x: A \vdash M: B}
\end{array}
$$

$\Pi$-FORM

$\left(s_{1}, s_{2}, s_{3}\right) \in \mathcal{R}$

$\frac{\Gamma \vdash A: s_{1} \quad \Gamma, x: A \vdash B: s_{2}}{\Gamma \vdash \Pi x: A . B: s_{3}}$

$\Pi$-INTRO

$\frac{\Gamma \vdash \Pi x: A . B: s \quad \Gamma, x: A \vdash M: B}{\Gamma \vdash \lambda x . M: \Pi x: A . B}$

$\Pi$-ELIM

$\frac{\Gamma \vdash M: \Pi x: A . B \quad \Gamma \vdash N: A}{\Gamma \vdash M N: B[N / x]}$
$\forall$-FORM

$$
\left(s_{1}, s_{2}, s_{3}\right) \in \mathcal{R}
$$

$\frac{\Gamma \vdash A: s_{1} \quad \Gamma, x: A \vdash B: s_{2}}{\Gamma \vdash \forall x: A . B: s_{3}}$

$\forall$-INTRO

$x \notin F V(M)$

$\frac{\Gamma \vdash \forall x: A . B: s \quad \Gamma, x: A \vdash M: B}{\Gamma \vdash M: \forall x: A . B}$

$\forall$-ELIM

$\frac{\Gamma \vdash M: \forall x: A . B \quad \Gamma \vdash N: A}{\Gamma \vdash M: B[N / x]}$

Conv

$\frac{\Gamma \vdash M: A \quad \Gamma \vdash B: s \quad A={ }_{\beta} B}{\Gamma \vdash M: B}$

Fig. 3. Typing rules for IPTS. Note that $\forall$-INTRO and $\forall$-ELIM are not syntax-directed.

are RESET, where Lemma 1 is used, and VAR and WEAK, which case split on whether $\Delta$ is empty. Corollary 3 is an immediate consequence of Lemma 2 .

Next, we prove the Substitution Lemma (4). Note that the mode $\tau_{1}$ of the typing judgment for the term $N$ to be substituted must match the context entry mark of the variable $x$ for which it will be substituted. Proof: By induction on the typing derivation. The interesting cases are REsET (requiring Corollary 3) and VAR and WEAK (each proceeding by cases on whether $\Delta=\varepsilon$ or not).

The Coherence Lemma (5) says that our type system is internally coherent in the following way - If it can prove that $M$ has type $A$, then it can also prove that $A$ is a type. Proof: By structural induction on the typing derivation. The interesting cases are RESET, which uses Lemma 1, and $\Pi$-ELIM, which makes use of Corollary 3 and Lemma 4.

Finally, Subject Reduction (Lemma 6) tells us that evaluation preserves types. Note that the mode $\tau$ of the typing judgment is preserved as well as the type. Proof: By structural induction on the typing derivation. The most interesting case is $\Pi$-ELIM in which we use Lemma 4.

\section{Implicit Pure Type Systems}

IPTS, the target language of the erasure translation, is an implicitly typed (Curry-style) calculus with both explicit and implicit dependent products. This 
calculus is modeled after Miquel's Implicit Calculus of Constructions (ICC) 1413, a Curry-style variant of Luo's Extended Calculus of Constructions [10]. ICC has a rich notion of subtyping that orders Church encodings at various levels of type refinement in a natural way 13 .

IPTS is both more and less general than ICC. It is more general because IPTS is defined in terms of an arbitrary PTS specification. It is less general because ICC (1) uses $\beta \eta$-conversion instead of $\beta$-conversion in determining type equality, (2) supports a notion of universe subtyping called cumulativity as in Luo's Extended Calculus of Constructions [10, and (3) contains extra typing rules ensuring $\eta$ subject reduction and strengthening.

The syntax of IPTS is as follows:

$$
\begin{aligned}
& \text { (terms) } \quad M, N, A, B::=x|\lambda x . M| M N|\Pi x: A . B| \forall x: A . B \mid s \\
& \text { (contexts) } \quad \Gamma, \Delta::=\varepsilon \mid \Gamma, x: A
\end{aligned}
$$

Note the distinction between $\Pi x: A . B$ and $\forall x: A . B$ as well as the omission of domain labels from $\lambda$-abstractions.

The difference between explicit and implicit products shows up in the type system (Figure 3). Whereas the explicit product is introduced by functional abstraction (rule $\Pi$-INTRO) and eliminated by function application (rule $\Pi$ ELIM), no syntactic cues indicate introduction or elimination of the implicit product (rules $\forall$-INTRO and $\forall$-ELIM). So $\Pi$ indicates functional abstraction and $\forall$ indicates polymorphism.

\section{Erasure}

We now define erasure as a translation from EPTS to IPTS.

$$
\begin{aligned}
& \text { Definition (Erasure). } \Gamma^{\bullet} \text { and } M^{\bullet} \\
& \varepsilon^{\bullet}=\varepsilon \quad\left(\Gamma, x:^{\tau} A\right)^{\bullet}=\Gamma^{\bullet}, x: A^{\bullet} \quad x^{\bullet}=x \quad s^{\bullet}=s \\
& \left(\Pi^{\mathrm{r}} x: A \cdot B\right)^{\bullet}=\Pi x: A^{\bullet} \cdot B^{\bullet} \quad\left(\lambda^{\mathrm{r}} x: A \cdot M\right)^{\bullet}=\lambda x \cdot M^{\bullet} \quad\left(M @^{\mathrm{r}} N\right)^{\bullet}=M^{\bullet} N^{\bullet} \\
& \left(\Pi^{\mathrm{c}} x: A \cdot B\right)^{\bullet}=\forall x: A^{\bullet} \cdot B^{\bullet} \quad\left(\lambda^{\mathrm{c}} x: A \cdot M\right)^{\bullet}=M^{\bullet} \quad\left(M^{\mathrm{c}} N\right)^{\bullet}=M^{\bullet}
\end{aligned}
$$

The bottom half of Figure 4 sketches out the meta-theory of erasure. We now discuss the significance of the results listed there.

A pair of key lemmas (7 and 8) characterize which variable occurrences in a term may survive erasure: those which are tagged with $r$ in the typing context. Proofs: Lemmas 7 and 8 must be proved simultaneously by structural induction on typing derivations. The WEAK case of the proof of Lemma 7 requires Lemma 8 and the $\Pi$-InTRo case of the proof of Lemma 8 requires Lemma 7 .

Preservation of Reductions. Since computation happens by substitution, we first show that erasure commutes with substitution (Lemma 9). We then 


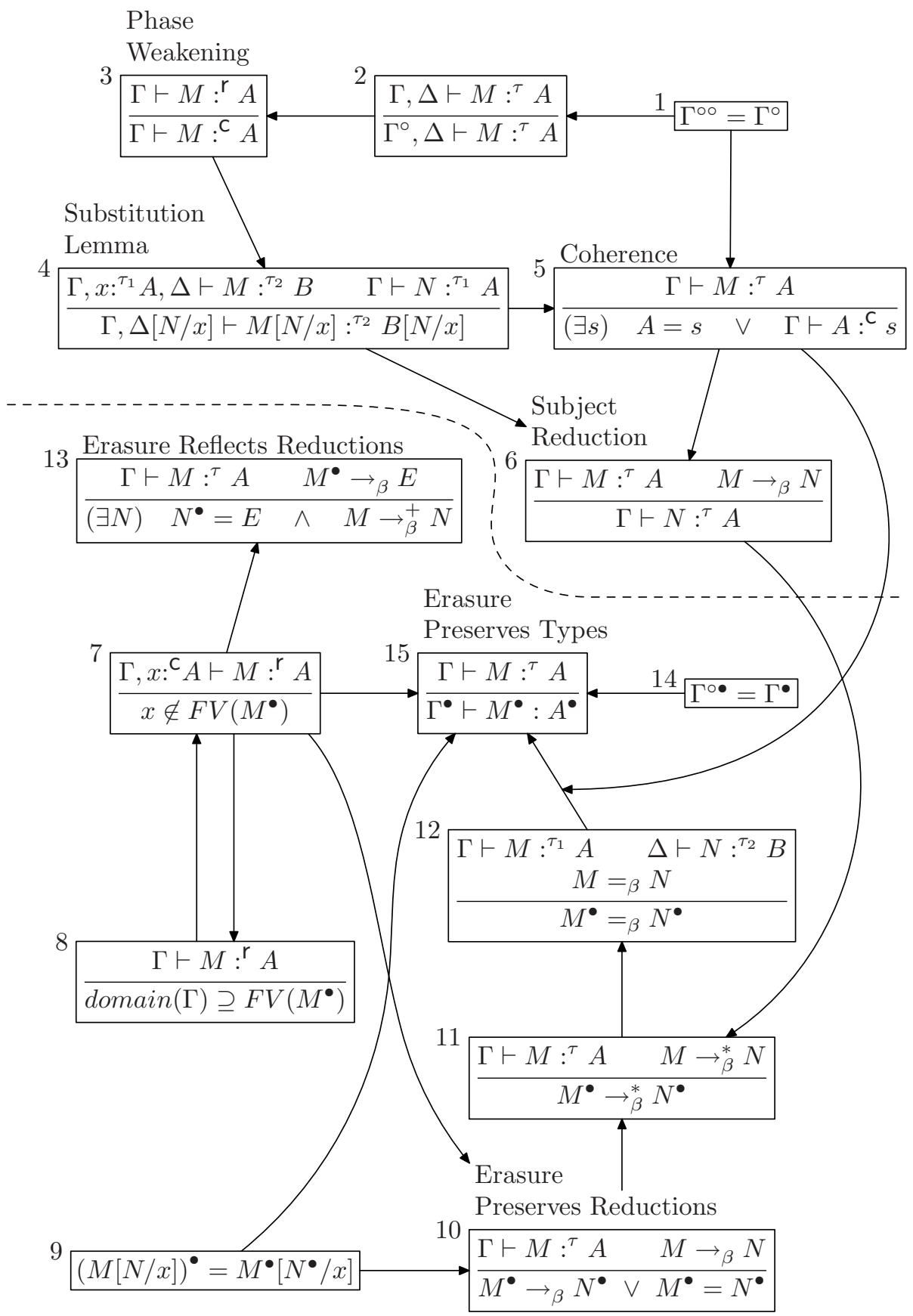

Fig. 4. Identities and admissible rules in the meta-theory of EPTS (above the dotted line) and erasure (below it). Arrows indicate proof dependencies. 
show that erasure respects reduction in the following sense: Each reduction step of a well-formed term in EPTS maps to either one or zero reduction steps in IPTS (Theorem 10). Proof: Lemma 9 is proved by straightforward induction on $M$. Theorem 10 is by straightforward induction over the typing derivation. The interesting cases are $\Pi$-INTRO and $\Pi$-ELIM, where we split by cases on $\tau$. In the $\Pi$-Elim case when the reduction step is $\beta$, the proof depends on Lemma 9 in the case where $\tau=\mathrm{r}$ and on Lemma 7 in the case where $\tau=\mathrm{c}$.

The proof of Theorem 10 shows that some EPTS reductions in fact do no work when viewed through the lens of erasure. This is precisely why we want an erasure semantics - to eliminate the work associated with run-time-irrelevant portions of a program. Examination of the proof shows where erasure eliminates work. As expected, the eliminated work includes erased redices (terms of the form $\left(\lambda^{\mathrm{C}} x: A . M\right) @^{\mathrm{c}} N$, which erase to just $\left.M^{\bullet}\right)$ as well as unnecessary reduction steps inside domain-annotations and erased arguments.

Corollaries 11 and 12 follow immediately from Theorem 10. The proof of Corollary 12 also requires the Church-Rosser Theorem.

Preservation of Typing. Again, we first investigate the properties of the context reset operation. The erasure operation annihilates it (Lemma 14). Proof: By induction on $\Gamma$.

Then we prove that erasure preserves well-typedness (Theorem 15). Proof: We prove Theorem 15 by structural induction on the typing derivation. The interesting cases are: RESET, in which Lemma 14 is used to simplify $\Gamma^{\circ} ; \Pi$ INTRO, in which Lemma 7 is used to ensure the premise $x \notin F V\left(M^{\bullet}\right)$ of the $\forall$-INTRO rule of IPTS; $\Pi$-ELIM, in which Lemma 9 is used to simplify the type of the application; and CONV, in which Coherence and Corollary 12 are used to establish the premise $A^{\bullet}={ }_{\beta} B^{\bullet}$ of the IPTS CONv rule.

Reflection of Reductions. Next we show that a reduction of a post-erasure IPTS term can be reflected back into one or more EPTS reductions (Theorem 13). Proof: By structural induction on the typing derivation. The interesting case is $\Pi$-ELIM when the @-annotation is $\tau=\mathrm{r}$ and the reduction is a $\beta$-step $\left(\lambda x . P^{\bullet}\right) N_{0}^{\bullet} \rightarrow{ }_{\beta} P^{\bullet}\left[N_{0}^{\bullet} / x\right]$. In this case, $M=M_{0} @^{r} N_{0}$ and $M_{0}^{\bullet}=\lambda x . P^{\bullet}$ and $E=P^{\bullet}\left[N_{0} \bullet x\right]$. The only way $M^{\bullet}$ can be $\lambda x . P^{\bullet}$ is if $M_{0}$ is a $\lambda^{\mathbf{r}} x: B$. $P$ nested under some (perhaps zero) "frames" of the form $\lambda^{\mathrm{C}} y: C$. [ ] or []@ ${ }^{\mathrm{C}} L$. Because the type of $M_{0}$ is $\Pi^{\mathrm{r}} x: A$. $B$, we know the top-most frame cannot be a $\lambda^{\mathrm{c}}$. Similarly, for typing reasons, the bottom-most frame cannot be a @ ${ }^{\mathrm{c}}$, because it is applied to a $\lambda^{r}$. Therefore, if there are any frames at all on top of $\lambda^{r} x: B$. $P$, then there are at least two, and at some point there is a $\lambda^{\mathrm{C}}$ frame just underneath a $@^{\mathrm{C}}$ one, forming a redex. If we reduce this redex, the rest of the frame structure remains intact. We repeat this process until no intermediate frames are left. Then $M_{0} \rightarrow_{\beta}^{*} \lambda^{r} x: B[\theta] . P[\theta]$ where $\theta$ is the simultaneous substitution effected by the sequence of reductions. Because $\theta$ is comprised solely of substitutions for $\lambda^{\mathrm{C}}$-bound variables, Lemma 7 tells us there will be no occurrences of these variables inside $P^{\bullet}$. Therefore $P[\theta]^{\bullet}=P^{\bullet}\left[\theta^{\bullet}\right]=P^{\bullet}$. Let $N=P[\theta]\left[N_{0} / x\right]$. Then

$$
N^{\bullet}=P[\theta]\left[N_{0} / x\right]^{\bullet}=P[\theta]^{\bullet}\left[N_{0}^{\bullet} / x\right]=P^{\bullet}\left[N_{0}^{\bullet} / x\right]=E
$$


and $M \rightarrow_{\beta}^{+} N$ because

$$
M=M_{0} @^{\mathbf{r}} N_{0} \rightarrow_{\beta}^{*}\left(\lambda^{\mathrm{r}} x: B[\theta] . P[\theta] @^{\mathrm{r}} N_{0} \rightarrow_{\beta} P[\theta]\left[N_{0} / x\right]=N,\right.
$$

thereby completing this case of the proof.

The proof of Theorem 13 shows that certain reduction steps in IPTS (of posterasure EPTS terms) require additional reductions in the original EPTS term before the reduction corresponding to that in IPTS can take place in EPTS. This means that some of the work that erasure avoids is unavoidable, in general, without erasure.

Theorem 13 says that any post-erasure reduction corresponds to some potential pre-erasure reductions. In other words, the erasure of a well-formed EPTS term cannot reduce in IPTS in a strange way that was not possible in EPTS.

\section{Erasure Semantics}

The erasure semantics for EPTS is simply this: First erase and then execute in IPTS. The meta-theory supports the claim that this is a good erasure semantics.

Theorem 10 : erasure eliminates some old work

Theorem 13 : erasure does not introduce any new work

Theorem 15 : erasure preserves the meanings (types) of programs

One final result supports the validity of our erasure semantics for EPTS. We would not want a PTS program to compute to a value while some annotation of it diverges under the erasure semantics. Thankfully, this cannot happen.

\section{Theorem (Erasure Preserves Strong Normalization)}

For a strongly normalizing PTS, any well-typed term in the corresponding EPTS erases to a strongly normalizing IPTS term.

Proof: Suppose there is an infinite reduction sequence in IPTS starting with the erasure of a well-typed term $M$ in EPTS. By Theorem 13 and Lemma 6, this reflects back into EPTS as an infinite reduction sequence starting with $M$. Because $b$ (the erasure-annotation-forgetting map from EPTS to PTS) preserves both reduction steps and typing judgments, we obtain an infinite reduction sequence in the underlying PTS starting with the well-typed term $M^{b}$. But this contradicts our assumption that the underlying PTS is strongly-normalizing.

\section{Implementation}

It should be easy to extend an existing type-checker to handle $\forall$-types. One must add $\tau$ annotations to the abstract syntax and some extra logic to the typechecker to handle these annotations properly. The only potential increase in the time complexity of type-checking comes from the context reset operation $\Gamma^{\circ}$. 
However, a clever representation of typing contexts renders reset a constanttime operation. The new representation of typing contexts is as follows:

$$
\Gamma::=(\hat{\Gamma} ; i) \quad \hat{\Gamma}::=\hat{\varepsilon} \mid \hat{\Gamma}, x:^{i} A
$$

(where $i$ denotes an integer). The context operations then become

$$
\begin{aligned}
& \varepsilon=(\hat{\varepsilon} ; 0) \quad \begin{array}{l}
(\hat{\Gamma} ; i), x:^{{ }^{C}} A=\left(\hat{\Gamma}, x:{ }^{i+1} A ; i\right) \\
(\hat{\Gamma} ; i), x:^{{ }^{\mathrm{r}} A}=\left(\hat{\Gamma}, x:^{i} A ; \quad i\right)
\end{array} \quad(\hat{\Gamma} ; i)^{\circ}=(\hat{\Gamma} ; i+1) \\
& x:{ }^{r} A \in(\hat{\Gamma} ; i) \text { iff } x:^{j} A \in \hat{\Gamma} \text { and } j \leq i
\end{aligned}
$$

The top-level $i$ in $\Gamma=(\hat{\Gamma} ; i)$ counts how many times prefixes of $\Gamma$ have been reset. For any binding $x:{ }^{j} A \in \hat{\Gamma}$ originally introduced with the mark $\tau$, we have $j>i$ iff (1) $\tau=\mathrm{c}$ and (2) there have been no resets since $x$ was introduced exactly the condition in which the binding for $x$ would be marked with $\mathrm{c}$ in the original implementation. The implementation of $x:{ }^{r} A \in \Gamma$ is therefore correct.

\section{Future Work}

\subsection{Proof Irrelevance}

In a dependently typed language, the conversion typing rule reflects the semantics of a language back into its type system. In EPTS, however, there are two notions of operational semantics. The CONV rule of EPTS reflects the default semantics rather than the erasure semantics. We may attempt to remedy this by modifying the CONV rule as follows:

$$
\frac{\mathrm{CONV}^{\bullet}}{\Gamma \vdash M:^{\mathrm{r}} A \quad \Gamma \vdash B:^{\mathrm{c}} s \quad A^{\bullet}={ }_{\beta} B^{\bullet}}
$$

This variant of EPTS is interesting because the $\mathrm{CONV}^{\bullet}$ rule seems to yield a generalized form of irrelevance, including proof irrelevance as a special case. Proof irrelevance in a conversion rule means that two proofs are considered equal if they prove the same proposition, regardless of how they each prove it. The $\mathrm{CONV}^{\bullet}$ rule only requires the run-time portions of $A$ and $B$ to be equal compile-time portions of $A$ and $B$ (including proofs and perhaps other terms) are considered irrelevant.

Pfenning's modal variant of LF with built-in notions of intensional code and proof irrelevance [17] provided inspiration for EPTS. The conversion rule of that system seems to us quite similar to $\mathrm{CONV}^{\bullet}$.

\subsection{Parametricity}

Languages such as Haskell and ML make heavy use of parametric polymorphism centered around a $\forall$ type constructor. Parametricity is a property of such languages enabling one to derive "free theorems" about polymorphic terms based 
solely on their type [21]. We conjecture that the $\forall$-types of EPTS satisfy parametricity properties similar to those of System F.

Many studies of parametricity for System F are based on denotational semantics. It seems impossible to develop a semantic model for an arbitrary EPTS. We think a proof-theoretic approach is necessary, somehow generalizing existing work on System F [18211].

\section{Conclusions}

Languages combining dependent types with erasure semantics sometimes require users to maintain more than one copy of a datatype in order to ensure erasure of some of its values but not others. This problem stems from the treatment of erasability as an intrinsic property of data, rather than a property of the way that data is used.

By treating erasability extrinsically — distinguishing functions that don't depend computationally on their arguments from those that do - we overcome the code duplication problem and arrive at a general form of polymorphism over arbitrary sorts of entities (types, proofs, numbers, etcetera).

This change of perspective leads to a notion of erasure generalizing both type-erasure and proof-erasure (program-extraction). We hope the resulting notion of computational irrelevance similarly generalizes both proof-irrelevance and parametricity-style notions of representation independence.

Acknowledgments. Thanks to Andrew Tolmach, Mark Jones, Jim Hook, Tom Harke, Chuan-Kai Lin, Ki-Yung Ahn, Andrew McCreight, Dan Brown, and John McCall for their comments on this work. This work is supported by the National Science Foundation (Grants Nos. CCF-0541447 and CCF-0613969).

\section{References}

1. The Coq proof assistant, http://coq.inria.fr

2. Abadi, M., Cardelli, L., Curien, P.-L.: Formal parametric polymorphism. Theoretical Computer Science 121(1-2), 9-58 (1993)

3. Augustsson, L.: Cayenne - A language with dependent types. In: Proceedings of the Third ACM SIGPLAN International Conference on Functional Programming, pp. 239-250 (1998)

4. Barendregt, H.P.: Lambda calculi with types. In: Abramsky, S., Gabbay, D.M., Maibaum, T.S.E. (eds.) Handbook of Logic in Computer Science, vol. 2, Oxford University Press, Oxford (1992)

5. Blazy, S., Dargaye, Z., Leroy, X.: Formal verification of a C compiler front-end. In: Misra, J., Nipkow, T., Sekerinski, E. (eds.) FM 2006. LNCS, vol. 4085, pp. 460-475. Springer, Heidelberg (2006)

6. Brady, E.: Practical Implementation of a Dependently Typed Functional Programming Language. PhD thesis, University of Durham (2005)

7. Chen, C., Xi, H.: Combining programming with theorem proving. In: Proceedings of the Tenth ACM SIGPLAN International Conference on Functional Programming, pp. 66-77 (2005) 
8. Leroy, X.: Formal certification of a compiler back-end, or: programming a compiler with a proof assistant. In: Proceedings of the 33rd ACM SIGPLAN Symposium on Principles of Programming Languages, pp. 42-54 (2006)

9. Lin, C., McCreight, A., Shao, Z., Chen, Y., Guo, Y.: Foundational typed assembly language with certified garbage collection. In: First Joint IEEE/IFIP Symposium on Theoretical Aspects of Software Engineering, pp. 326-338. IEEE Computer Society Press, Los Alamitos (2007)

10. Luo, Z.: Computation and reasoning: A type theory for computer science. Oxford University Press, New York, USA (1994)

11. Mairson, H.G.: Outline of a proof theory of parametricity. In: Hughes, J. (ed.) FPCA 1991. LNCS, vol. 523, pp. 313-327. Springer, Heidelberg (1991)

12. McBride, C., McKinna, J.: The view from the left. Journal of Functional Programming 14(1), 69-111 (2004)

13. Miquel, A.: The implicit calculus of constructions. In: Abramsky, S. (ed.) TLCA 2001. LNCS, vol. 2044, pp. 344-359. Springer, Heidelberg (2001)

14. Miquel, A.: Le Calcul des Constructions Implicite: Syntaxe et Sémantique. PhD thesis, Université Paris 7 (2001)

15. Necula, G.C.: Proof-carrying code. In: Proceedings of the 24th ACM SIGPLAN Symposium on Principles of Programming Languages, pp. 106-119 (1997)

16. Peyton-Jones, S., Vytiniotis, D., Weirich, S., Washburn, G.: Simple unificationbased type inference for GADTs. In: Proceedings of the Eleventh ACM SIGPLAN International Conference on Functional Programming (2006)

17. Pfenning, F.: Intensionality, extensionality, and proof irrelevance in modal type theory. In: LICS 2001: Proceedings of the 16th Annual Symposium on Logic in Computer Science, pp. 221-230. IEEE Computer Society Press, Los Alamitos (2001)

18. Plotkin, G.D., Abadi, M.: A logic for parametric polymorphism. In: Bezem, M., Groote, J.F. (eds.) TLCA 1993. LNCS, vol. 664, pp. 361-375. Springer, Heidelberg (1993)

19. Sheard, T.: Languages of the future. In: Proceedings of the Nineteenth ACM SIGPLAN Conference on Object-Oriented Programming, Systems, Languages, and Applications, OOPSLA Companion Volume, pp. 116-119 (2004)

20. Sheard, T., Pašalić, E.: Meta-programming with built-in type equality. In: Proceedings of the Fourth International Workshop on Logical Frameworks and MetaLanguages (LFM 2004), pp. 106-124 (2004), http://cs-www.cs.yale.edu/homes/carsten/lfm04/

21. Wadler, P.: Theorems for free! In: Functional Programming Languages and Computer Architecture, pp. 347-359. ACM Press, New York (1989)

22. Xi, H., Pfenning, F.: Dependent types in practical programming. In: Proceedings of the 26th ACM SIGPLAN Symposium on Principles of Programming Languages, pp. 214-227 (1999) 